\title{
Line-blanketing in massive stars: new results
}

\author{
D. John Hillier \\ Department of Physics and Astronomy, University of Pittsburgh, \\ 3941 O'Hara Street, Pittsburgh, PA, 15260, USA
}

\begin{abstract}
Until recertly, a major deficiency of most spectroscopic modeling of hot stars was the neglect of line-blanketing. With the availability of new atomic data, faster computers, suitable approximations, and improved numerical techniques it is now feasible to include non-LTE line-blanketing due to CNO and iron group elements in atmospheric calculations. We highlight the capabilities of the present generation of models. New blanketing results are presented for WN and WC Wolf-Rayet stars, and for LBVs. Line-blanketing alters individual line strengths in unpredictable ways, and can significantly influence the determination of stellar parameters. We specifically highlight the UV spectral region in which the effects of Fe blanketing are directly manifested. The importance of the charge exchange reaction $\mathrm{Fe}^{2+}+\mathrm{H} \rightleftharpoons \mathrm{Fe}^{+}+\mathrm{H}^{+}$for modeling of the $\mathrm{Fe}$ emission lines in the extreme P-Cygni star HDE 316285 is illustrated.
\end{abstract}

\section{Introduction}

The modeling of the spectra of hot stars is extremely complex. Since their atmospheres are extended the simplest geometry that can be adopted is spherical geometry. Further, due to the low densities and high UV fluxes, the use of nonLTE calculations is mandatory. Finally, in many hot stars the bulk of the flux is emitted shortward of the Lyman limit and hence is not directly observable. The Lyman continuum, generally responsible for driving the flow and determining the ionization structure, is heavily blanketed by bound-bound transitions which must be allowed for if realistic models are to be constructed.

Over the last two decades there has been significant progress in the spectroscopic modeling of the spectra of hot stars and their associated winds. Only recently has the goal of allowing for non-LTE line-blanketing been achieved (e.g., Dreizler \& Werner 1993; Schaerer \& Schmutz 1994; Hubeny \& Lanz 1995; Hauschildt et al. 1996; Hillier \& Miller 1998). It is now feasible to model the spectra of hot stars from the UV through to IR wavelengths.

Below we examine recent progress towards non-LTE calculations for several different classes of massive stars. We consider the WC5 star HD 165763, the Ofpe/WN star R 136a3, and the extreme P-Cygni star HDE 316285 (suspected LBV). Blanketed models for HD 96584 (WN8) are illustrated elsewhere in these Proceedings (Herald et al. 1999).

Further information on non-LTE line-blanketing calculations is given by Crowther (1999) and Pauldrach (1998). A review of recent progress in non-LTE line-blanketing calculations has been given by Hillier (1997b). 


\section{General comments}

\subsection{Continuum definition}

One of the difficulties of modeling the UV energy distribution of hot stars, and WR stars and LBVs in general, is that of locating the continuum. Most analyses have been done by normalizing the spectra to unity. For optical spectra of $\mathrm{O}$ stars (and to a lesser extent WN stars) this is straightforward since the continuum is fairly easy to define. In WC stars, however, the continuum is almost completely masked from view by the numerous $\mathrm{C}$ and $\mathrm{O}$ emission lines.

In the UV the continuum is difficult to define in most early-type stars, particularly shortward of $1900 \AA$. Often the true continuum cannot be identified with any assurity (see Figures 1, 2, and 3). Consequently it is very difficult to deduce reliable line strengths. This has important consequences for determining abundances of $\mathrm{Fe}$ group elements.

The advantage of blanketing models is that it is feasible to model the entire energy distribution, allowing the observed and predicted spectrum to be compared in all their fine details. Line blends, and the masking of the continuum by overlapping emission lines, are automatically allowed for with this procedure. However, there are two major problems with this approach.

First, for comparison with models and to allow precise continuum definition, accurate spectrophotometry (ideally 1\%) from IR to UV wavelengths is desirable. With care this can be achieved with current instrumentation, although errors quoted are typically $10 \%$.

Second, the light from massive stars generally suffers significant interstellar extinction. Unfortunately the UV extinction law is not known with great accuracy, and more importantly, it is known to vary with sightline. For some of the stars to be discussed below (e.g., HD 165763) there is evidence that the UV extinction law is not normal. In such cases it is feasible to derive the UV extinction law from a comparison of observations with the model. However, this does not allow the energy distribution predicted by the models to be tested an essential requirement if we are to have confidence in our results.

A further problem in the UV, particularly shortward of $1500 \AA$, is the presence of numerous discrete interstellar absorption features due to neutral $\mathrm{H}, \mathrm{H}_{2}$, $\mathrm{CO}$, and other atomic species. The $\mathrm{H}_{2}$ spectrum is particularly rich shortward of $1200 \AA$ and must be taken into account when modeling the UV spectrum (e.g., McCandliss et al. 1993; Taresch et al. 1997). High resolution observations can greatly assist in the modeling of the interstellar absorption features.

LMC O-type stars, with their low reddening, are particularly useful. Unfortunately, in the analysis of LMC stars there are three potential extinction laws to consider - a LMC extinction law, a 30 Doradus extinction law, and the galactic extinction law.

The severity of the reddening problem can be noted using the standard extinction law of Cardelli et al. (1988), which gives $A_{1200} / E_{B-V}=11.0$. Thus even if $E_{B-V}$ is known to an accuracy of 0.01 , the corresponding flux error at $1200 \AA$ is $10 \%$. More importantly, the differential reddening between $1100 \AA$ and $1600 \AA$ is $5 E_{B-V}$ (Cardelli et al. 1988) which can further exacerbate the difficulty of defining the continuum over this wavelength range. 


\subsection{Parameter space}

The advent of blanketing models and the inclusion of metals has significantly increased the size of the model parameter space to be explored. For example, a typical model of a WN/Ofpe star might be characterized by five parameters: $T_{\text {eff }}, \mathrm{N}(\mathrm{H}) / \mathrm{N}(\mathrm{He}), \mathrm{N}(\mathrm{Fe}) / \mathrm{N}(\mathrm{He}), \dot{M}^{2} / R_{*}{ }^{3}$ (wind density parameter, Schmutz et al. 1989), and $\beta$ (parameter characterizing the shape of the velocity law). Allowing each parameter to take on 5 values would necessitate the computation of 3125 models! Such a large grid is presently not feasible - especially since the models are still undergoing improvement. Furthermore, there are other parameters which can also be important $[e . g ., \mathrm{N}(\mathrm{N}) / \mathrm{N}(\mathrm{He}), \mathrm{N}(\mathrm{C}) / \mathrm{N}(\mathrm{He})]$.

While some abundances may only have a minor influence on the structure, and can be determined independently of the grid calculation, others may influence important diagnostic lines. This can occur directly (through blends) or indirectly through changes in the ionization structure. For example, for the WN8 star HD 96548 the inclusion of Si resulted in a 10\% change in the strength of the important diagnostic line He II $\lambda 4686$ (Herald et al. 1999).

The modeling of hot star spectra is not simply a matter of using a black box - physical insight and careful judgments about the models, the model quality, and the data quality are necessary.

\subsection{Influence of line-blanketing}

Due to the increased opacity provided by lines it is generally expected that there will be a redistribution of flux from the most heavily blanketed region to longer wavelengths. This expectation is generally borne out by the calculations.

With regards to line strengths no general predictions can be made. For simple recombination lines no changes may be observed provided the blanketing has not significantly altered the ionization structure. For lines formed via continuum fluorescence the changes in line strength may go in either direction. The importance of the fluorescence mechanism is strongly dependent on the UV flux, which can be reduced or enhanced by blanketing, at the wavelength of the pumping transition. A wide range in the reaction of certain individual lines to line blanketing is seen in preliminary blanketing calculations (e.g., Hillier \& Miller 1998, 1999).

Preliminary calculations suggest that blanketing effects lead to higher luminosities for both WCE and WNE stars. For example, Hillier \& Miller (1999) derived $L=2 \times 10^{5} \mathrm{~L}_{\odot}$ for HD 165763 (WC5), which is a factor of two higher than values previously determined using unblanketed calculations. For WNL stars (e.g., HD 96548) the luminosities do not appear to be substantially affected, although individual line strengths are modified (Herald et al. 1999).

\section{HD 165763 (WR 111, WC5)}

HD 165763 has been the subject of several non-LTE studies (Hillier 1989; Hamann et al. 1992; Koesterke \& Hamann 1995) and IR recombination line analyses (Nugis 1982; Eenens \& Williams 1992), which all suggest that $N(C) / N(H e)$ is of the order of 0.4. A detailed blanketed study of HD 165763 has recently been undertaken by Hillier \& Miller (1999) confirming this abundance ratio. 

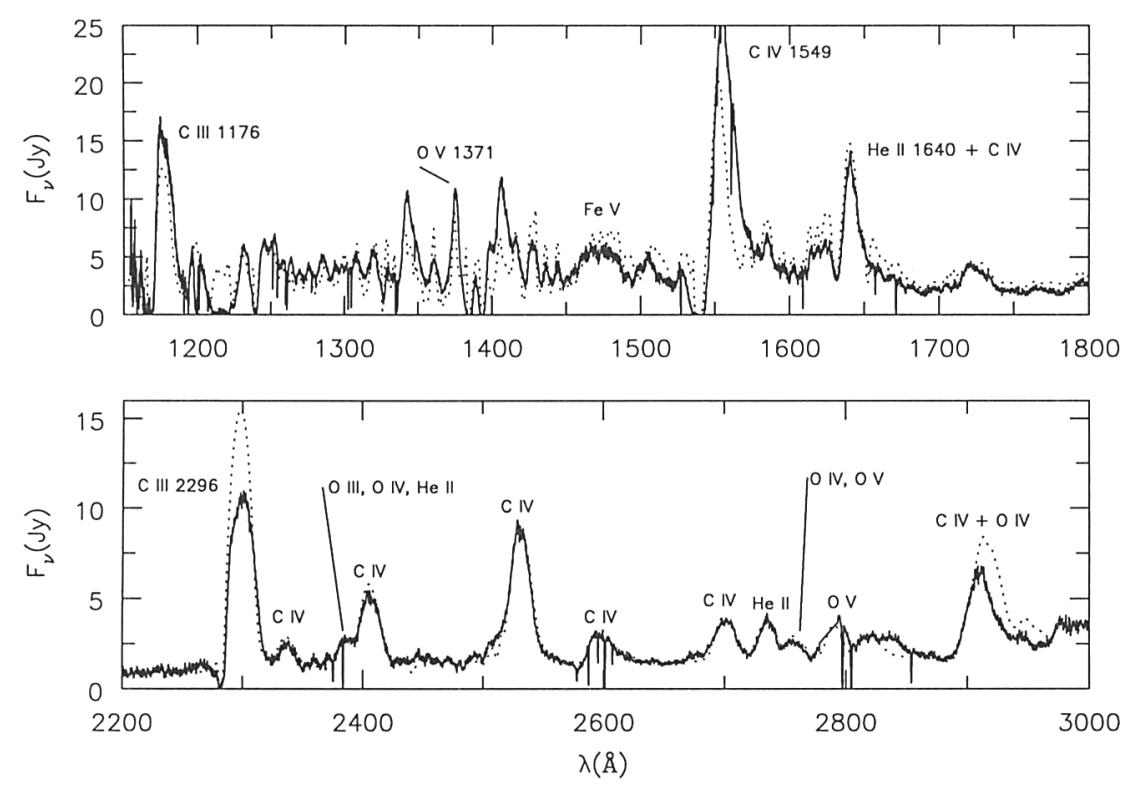

Figure 1. Comparison of the final model (dotted) of Hillier \& Miller (1999) with observations (solid) for HD 165763 (WC5). Major emission features are identified. Shortward of $1500 \AA$, Fe V and Fe VI emission/absorption lines permeate the spectrum.

The spectra of WC stars is difficult to model due to the richness of the emission line spectrum, the difficulty of locating the continuum, and the importance of several ionization stages of both $\mathrm{C}$ and $\mathrm{He}$ for defining the structure of the atmosphere. In early non-LTE calculations there were marked discrepancies between theory and observation. For example, the strength of the classification doublet, C IV $\lambda \lambda 5801,5812$, was underestimated by a factor of 3 (or more). Other lines (e.g., C III $\lambda 5696)$ were found to be extremely model sensitive.

With the advent of blanketed models the situation has improved considerably. It is now feasible to match the $\mathrm{C}_{\text {III }} \lambda \lambda 5801,5812$ doublet, although due to model sensitivities it is still very difficult to match this line simultaneously with the $\mathrm{C}$ III $\lambda 5696$ line. Importantly, the Fe lines in the UV can also be reproduced (e.g., Fe v complex near $1470 \AA$, Fig. 1).

Unfortunately, the reddening towards HD 165763 is significant $\left(E_{B-V}=0.3\right)$, and is possibly anomalous. We obtained an excellent fit to the flux spectrum from UV $(1200 \AA)$ to near-IR $(2.2 \mu \mathrm{m})$ using the extinction law of Cardelli et al. (1988) and an $\mathrm{R}$ value of 4.5. It is important that this high $\mathrm{R}$ value be independently verified.

A common problem with modeling of both WN and WC stars is that the electron scattering wings seen on strong emission lines are significantly stronger in the models than in the observations. The simplest explanation is to assume that the winds are clumped (e.g., Hillier 1991). Simple clumped models for WN stars have been considered by both Hillier (1991) and Schmutz (1997), and do lead to better agreement of model profiles with observations. 
To facilitate the calculations of line profiles in clumped WR winds, Hillier (1997) and Hillier \& Miller (1999) utilized a simple filling factor approach, and found (not surprisingly) that good fits to the spectra can be derived assuming a clumped wind. For HD 165763, Hillier \& Miller (1999) obtained $\dot{M}=5.0 \times$ $10^{-5} \sqrt{f}$ where $f$ is the volume filling factor. Good fits were found to the red wings of the profiles using $f=0.1$, although because of the severe blending in WC stars an accurate value of $f$ cannot (easily) be derived. The scaling of $\dot{M}$ with $f$, which simply reflects the importance of density-squared processes in WR atmospheres, was first derived by Abbott et al. (1981) who considered the influence of clumping on radio fluxes.

\section{R 136a3}

$\mathrm{R} 136 \mathrm{a}$, the core of the giant $\mathrm{H}$ II region 30 Doradus, is of extreme importance due to its use as a template for studying more distant starbursts. In conjunction with Alex de Koter, Sally Heap, and Ivan Hubeny, we have recently begun to model the star R 136a3 (Ofpe/WN) using line-blanketing calculations. The purpose of the initial analysis is two-fold. First, we wish to understand the reason for the discrepancy between the Hydrogen abundances deduced by de Koter et al. (1997; $\mathrm{He} / \mathrm{H}$ approximately solar) and that deduced by Crowther \& Dessart (1998; $\mathrm{N}(\mathrm{H}) / \mathrm{N}(\mathrm{He})=3)$. This difference has profound consequences for interpreting the nature of the massive stars in the $\mathrm{R} 136$ a cluster.

Second, we wish to perform a precise quantitative analysis of the most massive 30 Doradus stars using blanketing calculations. This will allow us to deduce Fe abundances, and will allow us to quantify the errors introduced by the use of non-line blanketed calculations for such stars. This work will form an excellent basis for the analysis of more distant starbursts.

In Fig. 2 we show theoretical spectra of three models with different effective temperature with parameters near those of $\mathrm{R} 136 \mathrm{a} 3\left(R_{*} \approx 18 \mathrm{R}_{\odot} ; \dot{M}=5.75 \times 10^{-5}\right.$ $\left.\mathrm{M}_{\odot} \mathrm{yr}^{-1} ; \mathrm{N}(\mathrm{H}) / \mathrm{N}(\mathrm{He})=3.25 ; \beta=1.5\right)$. Diagnostics of $T_{\text {eff }}$ can be clearly identified. Of course such diagrams do not allow a direct deduction of $T_{\text {eff }}$ : we also need to allow for variations in the other fitting parameters $(e . g ., \dot{M})$. Detailed fits of the models with observation will be presented elsewhere.

\section{HDE 316285}

HDE 316285 is an extreme P-Cygni star, and has recently been extensively studied using non-LTE non-blanketed models by Hillier et al. (1998). One of the striking aspects of its spectrum is the strong Fe II metal lines in the optical particularly multiplet no. $42(\lambda \lambda 4924,5018,5169)$, and the low ionization metal lines of $\mathrm{Mg}$ II, $\mathrm{AlII}, \mathrm{Al} I I I, \mathrm{NaI}$ which are produced by continuum fluorescence.

Initial line-blanketed model calculations showed that it was not possible to produce the strong P-Cygni multiplet no. 42 Fe II profiles with a solar massfraction of iron. As $\mathrm{H}$ can recombine in the wind of these stars (e.g., Najarro et al. 1997) it is important to allow for charge exchange reactions with neutral $\mathrm{H}$. Inclusion of the charge exchange reaction $\mathrm{Fe}^{2+}+\mathrm{H} \rightleftharpoons \mathrm{Fe}^{+}+\mathrm{H}^{+}$(Neufeld \& Dalgarno 1987) is found to have a major influence on the Fe ionization struc- 

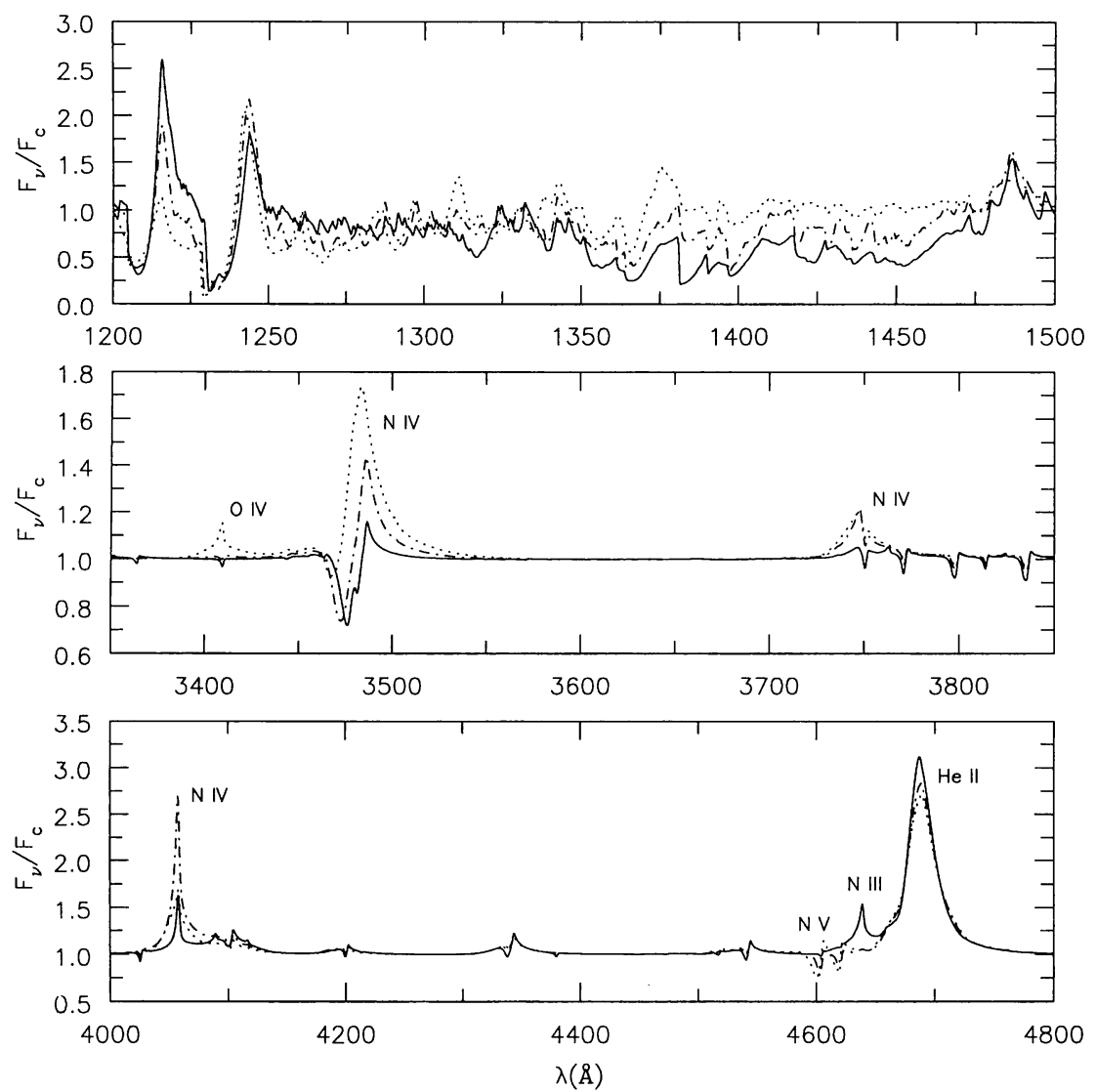

Figure 2. Illustration of the effect of changes in $T_{\text {eff }}$ on the spectrum of a star with parameters similar to $\mathrm{R} 136 \mathrm{a} 3$. Shown are theoretical spectra for $T_{\text {eff }}=38,000 \mathrm{~K}$ (solid line), $T_{\text {eff }}=44,500 \mathrm{~K}$ (dot-dash) and $T_{\text {eff }}=52,000 \mathrm{~K}$. The very broad absorption feature centered near $1400 \AA$ is due to a host of transitions primarily belonging to $\mathrm{FeV}$ and $\mathrm{Fe} \mathrm{VI}$. Because of the wealth of absorption/emission features it is extremely difficult to draw a reliable continuum between 1200 and $1500 \AA$.

ture, and significantly enhances the Fe II line strengths (Fig. 3). With the above reaction included the deduced Fe mass fraction is only twice solar.

For heavily reddened stars only the IR spectrum is available for spectroscopic analysis. HDE 316285 (and P-Cygni) provide a means of testing the validity of IR analyses using optical (and/or UV) lines (Najarro et al. 1999a, 1999b).

\section{Discussion}

While the tools necessary to undertake detailed non-LTE line-blanketing calculations have been developed, much work remains to be done in order to improve 

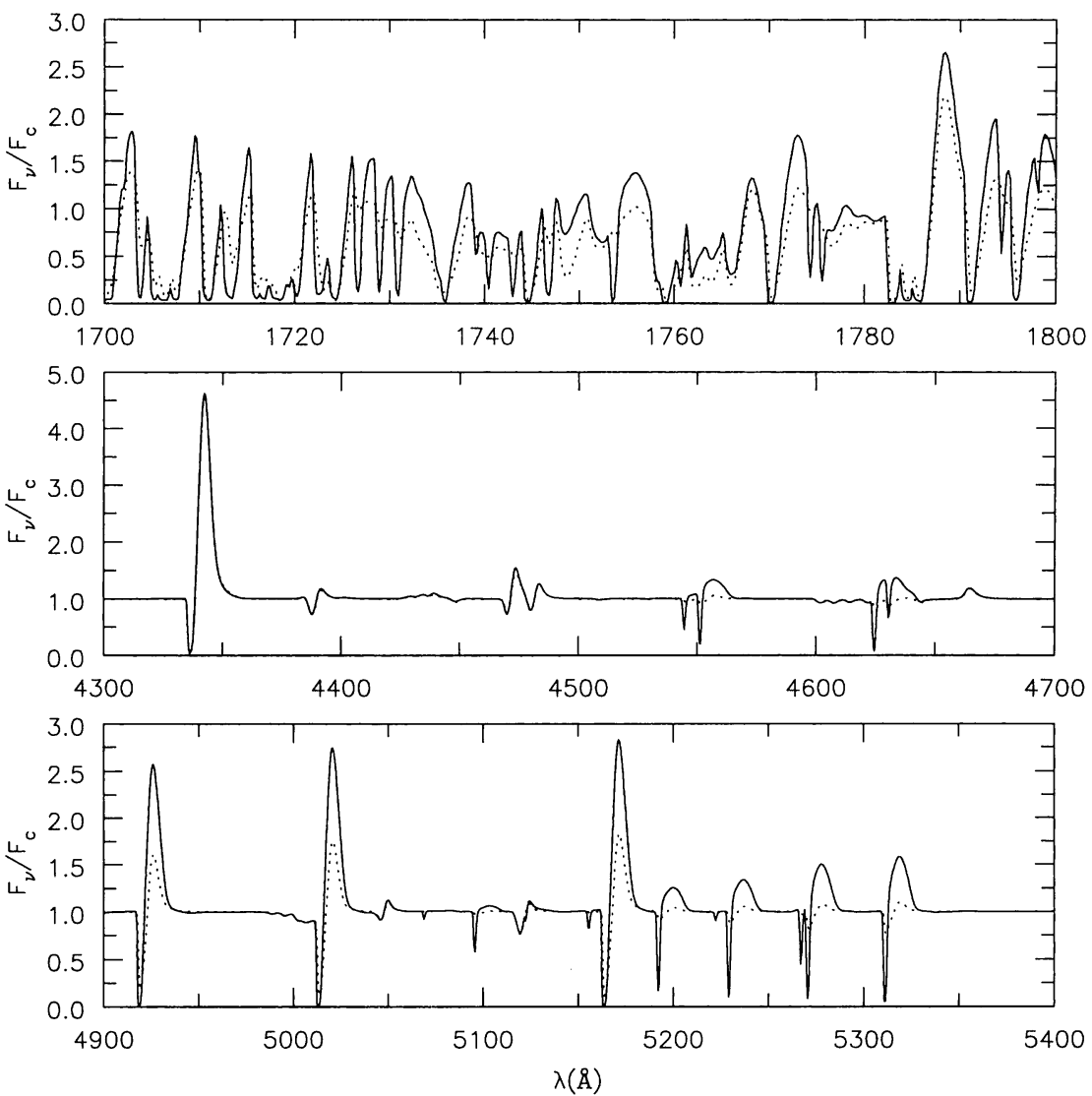

Figure 3. Illustration showing the effect of charge exchange reactions on the Fe II emission lines for a model with parameters similar to those of HDE $316285\left(R_{*}=75 \mathrm{R}_{\odot}, \dot{M}=2.0 \times 10^{-4} \mathrm{M}_{\odot} \mathrm{yr}^{-1}, T_{\text {eff }} \approx 15,000 \mathrm{~K}\right.$, $\left.L=2.75 \times 10^{5} \mathrm{~L}_{\odot}, v_{\infty}=410 \mathrm{~km} \mathrm{~s}^{-1}, \beta=2.5\right)$. The solid line shows the theoretical spectrum with charge exchange reactions included while the dotted line is the spectrum without charge exchange reactions. In the optical Fe II emission line strengths [e.g., multiplet no. $42(\lambda \lambda 4924,5018,5169)$; multiplet no. 49 $(\lambda \lambda 5198,5235,5276,5317)]$ are significantly enhanced. Most other emission lines (e.g., $\mathrm{H} \gamma \lambda 4340)$ are not affected. A comparison of the model spectrum with observations is given elsewhere in these Proceedings (Hillier et al. 1999). Note the extreme line-blanketing in the UV spectral region.

spectroscopic analyses of massive stars. First, more complete ions and better atomic data, particularly for the Fe group atoms are required. Second, in order to model winds, we need to understand their structure. Theoretical (e.g., Owocki et al. 1988) and observational work (e.g., Lepine \& Moffat 1999) has given important insights but many questions remain unanswered. In particular, how is the wind clumped? How does the clumping vary with position in the wind? The computation of spectra in highly structured winds is probably the 
largest hurdle left to overcome for reliable spectroscopic analysis of the spectra of single massive stars and their associated winds.

Observationally, better spectrophotometric data is required. Additionally, a better understanding of the reddening law and its behaviour is required. For example, how good is the one-parameter reddening law proposed by Cardelli et al. (1988)? Is one parameter sufficient to describe most reddening laws, or are additional parameters required? What is the reddening law in the EUV (i.e., shortward of $1200 \AA)$ ?

With the advent of line blanketed models we are in a position to deduce accurate stellar parameters and abundances. These will provide strong constraints on stellar evolution. Furthermore, with reliable Fe abundances, we will be better able to test stellar wind theory for all hot stars. These new blanketed atmospheric models will also provide significantly improved ionizing fluxes for modeling of $\mathrm{H}$ II regions.

Acknowledgments. Special thanks to my collaborators P.A. Crowther, S. Heap, J. Herald, A. de Koter, D. Miller, F. Najarro, R. Schulte-Ladbeck for their contributions to various aspects of the work presented above. This research was supported in part by NASA through grant numbers GO 4450.01-92A, GO 0662201-95A, and AR 07985.02-96A from the STScI, which is operated by AURA, under NASA contract NAS5-26555.

\section{References}

Abbott, D.C, Bieging, J.H., Churchwell, E. 1981, ApJ 250, 645

Crowther, P.A. 1999, in: K.A. van der Hucht, G. Koenigsberger \& P.R.J. Eenens (eds.), Wolf-Rayet Phenomena in Massive Stars and Starburst Galaxies, Proc. IAU Symp. No. 193 (San Francisco: ASP) (these Proceedings)

Crowther, P.A., Dessart, L. 1998, MNRAS 296, 622

Cardelli, J.A., Clayton, G.C., Mathis, J.S. 1988, ApJ 329, L33

Dreizler, S., Werner, K. 1993, A\&A 278, 199

Eenens, P.R.J., Williams, P.M. 1992, MNRAS 255, 227

Koesterke, L., Hamann, W.-R. 1995, A\&A 299, 503

de Koter, A., Heap, S.R., Hubeny, I. 1997, ApJ 477, 792

Hamann, W.-R., Leunenhagen, U., Koesterke, L., Wessolowski, U. 1992, A\&A 255, 200

Hauschildt, P.H., Baron, E., Starrfield, S., Allard, F. 1996, ApJ 462, 386

Herald, J., Schulte-Ladbeck, R.S., Hillier, D.J. 1999, in: K.A. van der Hucht, G. Koenigsberger \& P.R.J. Eenens (eds.), Wolf-Rayet Phenomena in Massive Stars and Starburst Galaxies, Proc. IAU Symp. No. 193 (San Francisco: ASP) (these Proceedings)

Hillier, D.J. 1989, ApJ 347, 392

Hillier, D.J. 1991, A\&A 247, 455

Hillier, D.J. 1997a, in: J. M. Vreux, A. Detal, D. Fraipont-Caro, E. Gosset, \& G. Rauw (eds.) Wolf-Rayet Stars in the Framework of Stellar Evolution, Proc. 33rd Liege International Astrophysical Colloquium, (Liége:Université de Liége), p. 509

Hillier, D.J. 1997b, in: T.R. Bedding, A.J. Booth \& J. Davis (eds.), Fundamental Stellar Properties: the Interaction between Observation and Theory, Proc. IAU Symp. No. 189 (Dordrecht: Kluwer), p. 209

Hillier, D.J., Crowther, P.A., Najarro, F., Fullerton, A.W. 1998, A\&A 340, 483 
Hillier, D.J., Crowther, P.A., Najarro, F., Fullerton, A.W. 1999, in: K.A. van der Hucht, G. Koenigsberger \& P.R.J. Eenens (eds.), Wolf-Rayet Phenomena in Massive Stars and Starburst Galaxies, Proc. IAU Symp. No. 193 (San Francisco: ASP) (these Proceedings)

Hillier, D.J., Miller, D.L. 1998, ApJ 496, 407

Hillier, D.J., Miller, D.L. 1999, ApJ in press

Dreizler, S., Werner, K. 1993, A\&A 278, 199

Hubeny, I., Lanz, T. 1995, ApJ 439, 875

Lepine, S., Moffat, A.F.J. 1999, ApJ 514, 909

McCandliss, S.R., Buss, R.H., Blair, W.P., et al. 1993, ApJ 416, 372

Najarro, F., Hillier, D.J., Stahl, O. 1997, A\&A 326, 1117

Najarro, F., Hillier, D.J., Figer, D.F. 1999a, in: K.A. van der Hucht, G. Koenigsberger \& P.R.J. Eenens (eds.), Wolf-Rayet Phenomena in Massive Stars and Starburst Galaxies, Proc. IAU Symp. No. 193 (San Francisco: ASP) (these Proceedings)

Najarro, F., Hillier, D.J., Figer, D.F., Geballe, T.R. 1999b, in: H. Falcke, A. Cotera, W. Duschl, F. Melia \& M. Rieke, The Central Parsecs of the Galaxy, ASP-CSin press

Neufeld, D.A., Dalgarno, A. 1987, Phys. Rev. A. 35, 3142

Nugis, T. 1982, in: C.W.H. de Loore \& A.J. Willis (eds.), Wolf-Rayet Stars: Observations, Physics, Evolution, Proc. IAU Symp. No. 99, (Dordrecht: Reidel), p. 127

Owocki, S.P., Castor, J.I., Rybicki, G.B. 1988, ApJ 335, 914

Pauldrach, A.W.A, Lennon, M., Hoffmann, T.L., Sellmaier, F., Kudritzki, R.P., Puls, J., 1998, in: I. Howarth (ed.), Boulder-Munich II, Properties of Hot Luminous Stars, ASP-CS 131, 258

Schaerer, D., Schmutz, W. 1994, A\&A 288, 231

Schmutz, W., Hamann, W.-R., Wessolowski, U. 1989, A\&A 210, 236

Schmutz, W. 1997, A\&A 321, 268

Taresch, G., Kudritzki, R.P., Hurwitz, M., et al. 1997, A\&A 321, 531

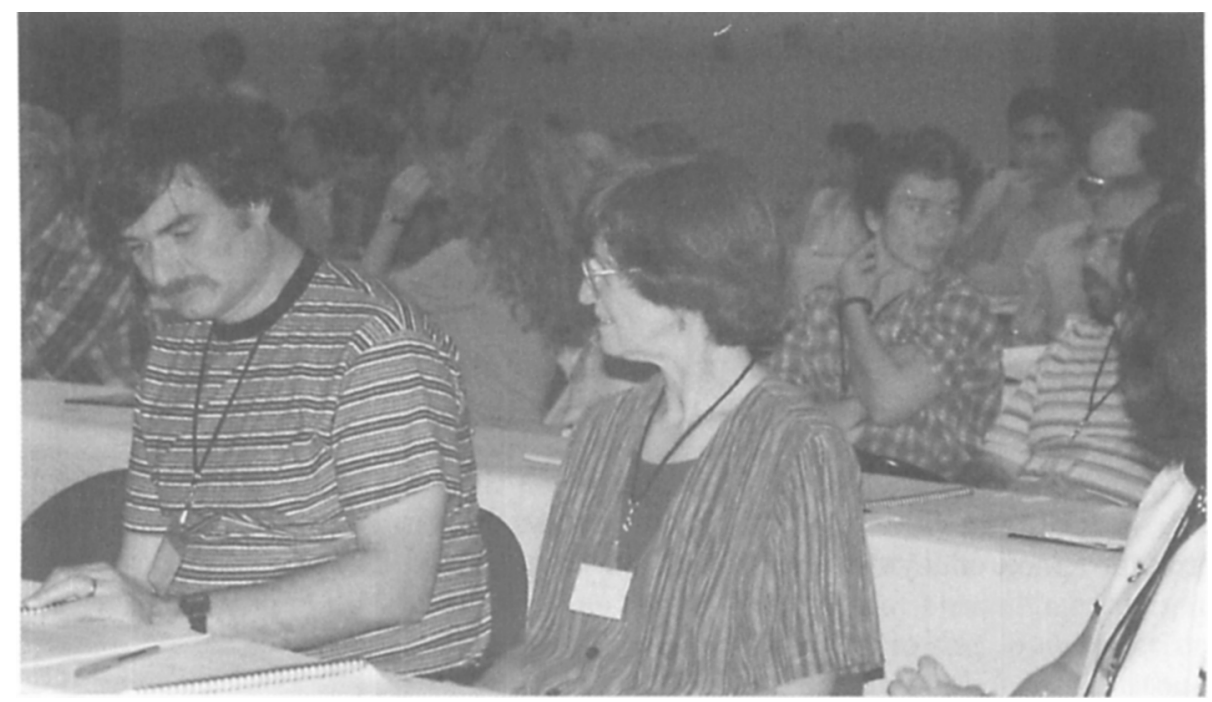

